\title{
Depletion of polycistronic transcripts using short interfering RNAs: cDNA synthesis method affects levels of non-targeted genes determined by quantitative PCR
}

\author{
Jennifer E Hanning, lan J Groves, Mark R Pett and Nicholas Coleman ${ }^{*}$
}

\begin{abstract}
Background: Short interfering RNAs (siRNAs) are often used to deplete viral polycistronic transcripts, such as those encoded by human papillomavirus (HPV). There are conflicting data in the literature concerning how siRNAs targeting one HPV gene can affect levels of other genes in the polycistronic transcripts. We hypothesised that the conflict might be partly explained by the method of cDNA synthesis used prior to transcript quantification.

Findings: We treated HPV16-positive cervical keratinocytes with siRNAs targeting the HPV16 E7 gene and used quantitative PCR to compare transcript levels of E7 with those of E6 and E2, viral genes located upstream and downstream of the target site respectively. We compared our findings from cDNA generated using oligo-dT primers alone with those from cDNA generated using a combination of random hexamer and oligo-dT primers. Our data show that when polycistronic transcripts are targeted by siRNAs, there is a period when untranslatable cleaved mRNA upstream of the siRNA binding site remains detectable by PCR, if CDNA is generated using random hexamer primers. Such false indications of mRNA abundance are avoided using oligo-dT primers. The period corresponds to the time taken for siRNA activity and degradation of the cleaved transcripts. Genes downstream of the siRNA binding site are detectable during this interval, regardless of how the cDNA is generated.

Conclusions: These data emphasise the importance of the cDNA synthesis method used when measuring transcript abundance following siRNA depletion of polycistronic transcripts. They provide a partial explanation for erroneous reports suggesting that siRNAs targeting HPV E7 can have gene-specific effects.
\end{abstract}

Keywords: Quantitative PCR, Short interfering RNA, CDNA synthesis

\section{Findings}

Gene depletion using siRNAs is an important tool in biological investigations and clinical therapeutics. It is often applied for depleting polycistronic transcripts, particularly those derived from viruses [1]. One example is high-risk human papillomavirus (HR-HPV), the necessary cause of cervical carcinoma [2]. Numerous siRNAs have been designed to target the major HR-HPV oncogenes E6 and E7. These genes are expressed in multiple polycistronic transcripts, in which E6 is upstream of E7 [3]. In cells where HR-HPV is integrated into host DNA the

\footnotetext{
* Correspondence: nc109@cam.ac.uk

Department of Pathology, University of Cambridge, Tennis Court Road, Cambridge CB2 1QP, UK
}

transcripts often contain E6 and E7 only, whereas in cells where the virus remains extra-chromosomal (episomal) there are additional downstream genes, including E2. There are conflicting reports regarding the effect of particular siRNAs on expression of other genes in the polycistronic transcripts, as assessed using quantitative reverse-transcription PCR (qRT-PCR). While some studies show that siRNAs targeting E7 also deplete E6 (and vice versa) [4,5], there is also widely-cited evidence that siRNAs can specifically deplete E7 without affecting E6 [6].

We hypothesised that the different results reported may be partly explained by the method used to generate 
Table 1 Sequences of siRNAs targeting HPV16 E7

\begin{tabular}{lll}
\hline siRNA source & Name & $\begin{array}{l}\text { siRNA sense (passenger) strand } \\
\text { sequence }\end{array}$ \\
\hline Tang [4] & Tang & GCACACACGUAGACAUUCG \\
Dharmacon & 127 & GGACAAGCAGAACCGGACA \\
Dharmacon & 141 & GGACAGAGCCCAUUACAAU \\
Dharmacon & 653 & GCUCAGAGGAGGAGGAUGA \\
Yamato [5] & Y573 & CACCUACAUUGCAUGAAUA \\
Jiang and Milner [6] & Jiang & AGGAGGAUGAAAUAGAUGG \\
\hline
\end{tabular}

CDNA prior to PCR quantification of individual HR-HPV oncogenes. Binding of siRNAs can result in cleaved transcripts that are subsequently degraded, at a rate that varies between systems [7]. While mRNA upstream of the cleavage site would not be translated, as it has no polyA tail, the genes encoded would still be detectable by PCR if cDNA was generated using random hexamer primers. In contrast, mRNA upstream of the cleavage site would not be detectable if cDNA was generated using oligo-dT primers, which would only generate cDNA from downstream of the cleavage site.

We initially tested this hypothesis by treating the HPV16-positive cervical squamous cell carcinoma (SCC) cell line CaSki [8] with siRNAs targeting HPV16 E7 and measuring levels of E7 and the upstream gene E6. We used six different siRNAs that mapped along the E7 open reading frame (Table 1 and Figure 1) [9]. Cells were transfected according to manufacturer's instructions using Lipofectamine RNAiMAX (Invitrogen, Paisley, UK) and $9 \mathrm{nM}$ siRNA. To control for non-specific siRNA effects, a pool of non-targeting control siRNAs (ON-TARGET plus non-targeting, Thermo Scientific) was used, also at 9nM. RNA was extracted using TRIzol (Life Technologies, Grand Island, NY, USA) according to manufacturer's instructions. Twenty-four hours after each treatment, cDNA was generated using two different methods: a combination of random hexamer and oligo-dT primers (QuantiTect reverse transcription kit, Qiagen, Crawley, $\mathrm{UK})$, and oligo-dT primers alone (Qiagen, UK). Levels of
HPV16 E7 and E6 were then measured by qRT-PCR as described [9], using the primers listed in Table 2. Primer binding sites are illustrated in Figure 1.

Across the siRNAs, the mean level of E7 depletion measured at 24 hours was $74.7 \%$ (range 59.2-84.2\%) for cDNA made with random hexamer and oligo-dT primers and $71.6 \%$ (46.1-84.8\%) for cDNA made with oligo-dT primers alone (Figure 2A). For cDNA made using oligo-dT primers only, levels of E6 mirrored those of E7 (Figure 2B). In contrast, for all six siRNAs, when using cDNA made with combined random hexamer and oligo-dT primers, E6 reductions were significantly lower than E7 (Figure 2B), with a mean reduction of $41.9 \%$ (range $31.8-50.8 \%) \quad(P$-value $=0.00011, \quad \mathrm{t}$-test $)$. Such differences between E6 and E7 levels were greatest for the siRNAs that produced the greatest depletion of E7, although the trend was maintained for all siRNAs.

The most potent siRNA, E7-141, was subsequently optimised for transfection in a different HPV16containing cervical squamous cell line, W12 series-4 passage-86 (W12Ser4p86) [10]. These cells reform an SCC in organotypic tissue culture and stably retain approximately 500 episomes of HPV16 per cell. They therefore express polycistronic transcripts in which E6 is upstream of E7, with other early genes including E2 downstream [10]. We predicted that levels of E6 depletion determined following cDNA synthesis using oligo-dT primers would be greater than those determined following cDNA synthesis using random hexamer primers but that depletion of E2 would be similar for both cDNA synthesis methods. Furthermore, measuring the disparities in viral gene levels over time would allow us to detect the interval over which siRNA activity and degradation of cleaved transcripts occurred. Accordingly, we measured of E7, E6 and E2 at 24, 48 and 96 hours following E7-141 treatment of W12Ser4p86.

As with CaSki, E7-141 treatment produced mean E7 depletion levels of $82.4 \%$, which did not vary significantly over the time-period studied and did not differ according to cDNA synthesis method (Figure 2C). At 24 hours following treatment the levels of E6 depletion

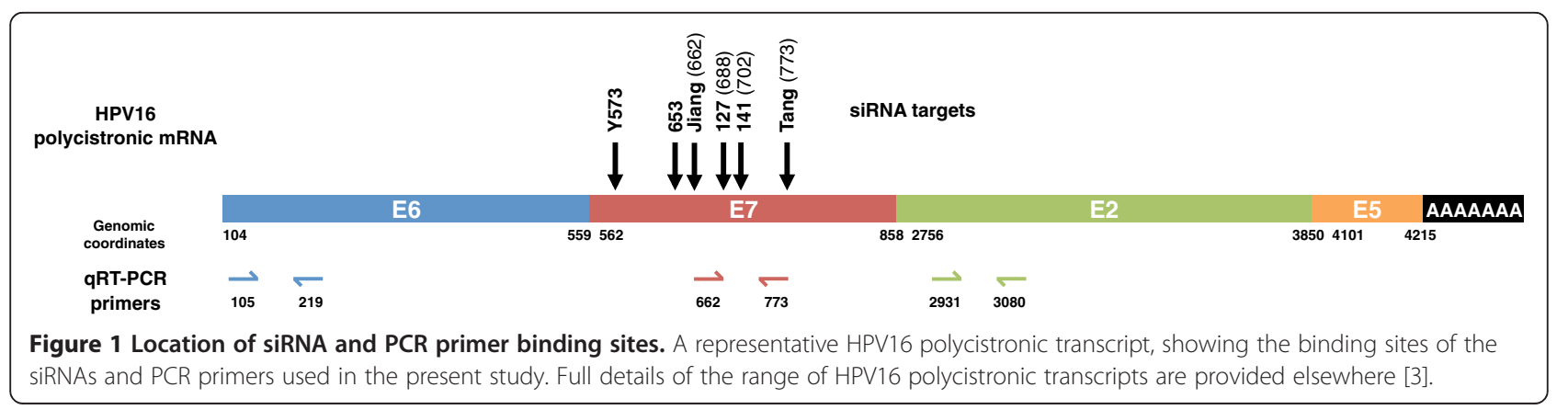


Table 2 Primers used for qRT-PCR

\begin{tabular}{lll}
\hline Target & Forward primer $\left(\mathbf{5}^{\prime}\right.$ to $\left.\mathbf{3}^{\prime}\right)$ & Reverse primer $\mathbf{( 5}^{\prime}$ to $\left.\mathbf{3}^{\prime}\right)$ \\
\hline HPV16 E7 & AGGAGGATGAAATAGATGGTCC & CTTGTACGCACAACCGAGC \\
HPV16 E6 6 -773) & AGCGACCCAGAAAGTTACCA & GCATAAATCCCGAAAAGCAA \\
(nt 105-219) & & CACATTCTAGGCGCATGTGT \\
HPV16 E2 & GGAGACTCTTGCCAACGTTA \\
(nt 2931-3080) & & GGCATGGACTGTGGTCATGAG \\
GAPDH & TGCACCACCAACTGCTTAGC \\
TBP & Hs_TBP_1_SG (Qiagen) & \\
ACTB & Hs_ACTB_2_SG (Qiagen) & \\
\hline
\end{tabular}

detected in cDNA generated using oligo-dT alone were similar to those determined for E7 (70.0\% for E6, 76.6\% for E7: Figure $2 \mathrm{C}$ and D), whereas the levels of E6 depletion determined in cDNA generated by random hexamers and oligo-dT were significantly less $(23.6 \%, P$-value $=$ 0.01359, t-test) (Figure 2D). Levels of E2 depletion at 24 hours were very low (mean $=4.9 \%$ ), regardless of the cDNA synthesis method (Figure 2E). In contrast, at 48 and 96 hours, levels of E6 and E2 depletion determined by PCR were similar to those of E7, regardless of the CDNA synthesis method (Figure 2C-E).

Our data are consistent with the model shown in Figure 3. Treatment with E7-targetting siRNAs leads to cleavage of the HPV16 polycistronic transcripts around the site of binding, preventing detection of the target gene E7 regardless of the mode of cDNA synthesis.
However, as siRNA activity and degradation of the cleaved transcripts progress, there is a window (between 24 and 48 hours in our W12 experiment) where misleading information may be obtained. For genes in the transcript that are upstream of the siRNA binding site (E6 in our experiments), PCR quantification using cDNA generated by random hexamers will detect untranslatable transcripts and so give an inappropriately high indication of gene abundance. A more appropriate measure of the abundance of such upstream genes would be provided by cDNA generated using oligo-dT primers alone. For genes in the polycistronic transcript that are downstream of the siRNA binding site (such as E2), cDNA generated using either random hexamers or oligo-dT primers would give a falsely high indication of gene abundance, until siRNA activity and degradation of the cleaved transcript is complete.
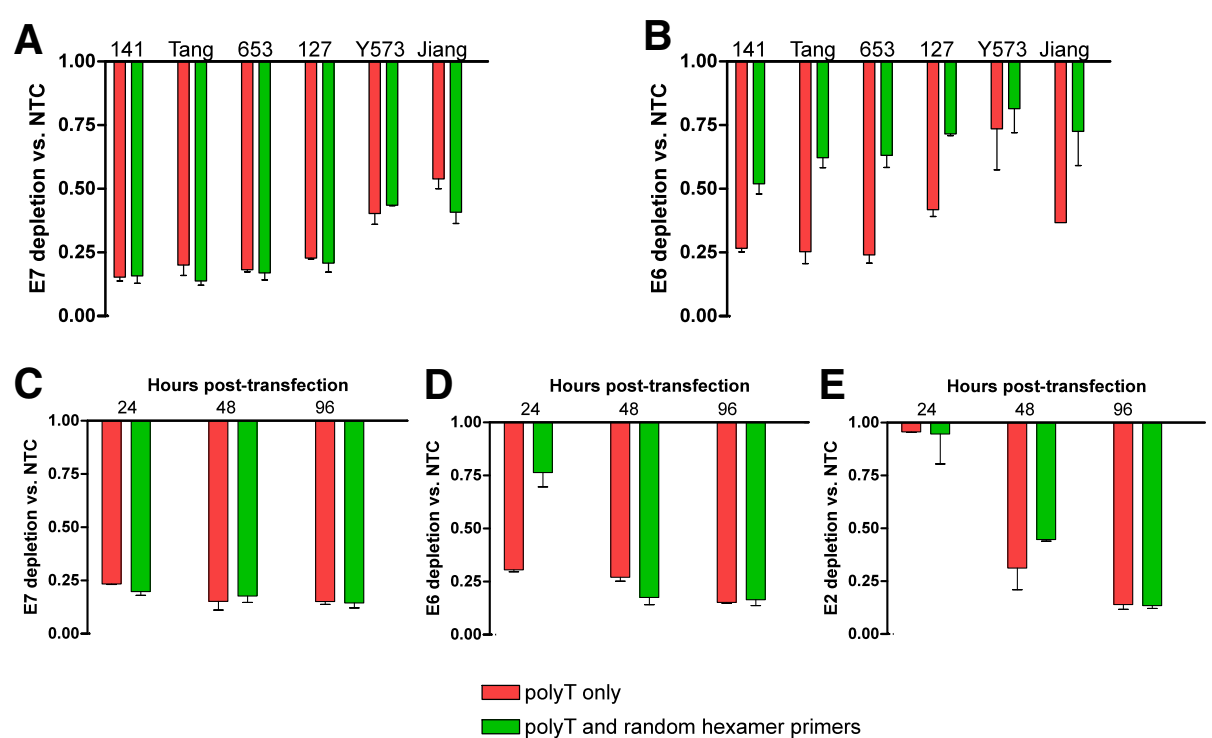

\footnotetext{
Figure 2 RT-PCR quantification of HPV16 mRNA depletion in E7-siRNA treated cells. The top row shows levels of A) E7 and B) E6 24 hours after treatment of CaSki with E7-targeting siRNAs. The bottom row shows levels of C) E7, D) E6 and E) E2 at 24, 48 and 96 hours after treatment of W12Ser4p86 with E7-141 siRNAs. Each pair of bars shows levels detected in the same cells using CDNA generated by oligo-dT primers only (red) or oligo-dT and random hexamer primers (green). Error bars show the standard deviations, based on three independent experiments. NTC = non-targeting control.
} 


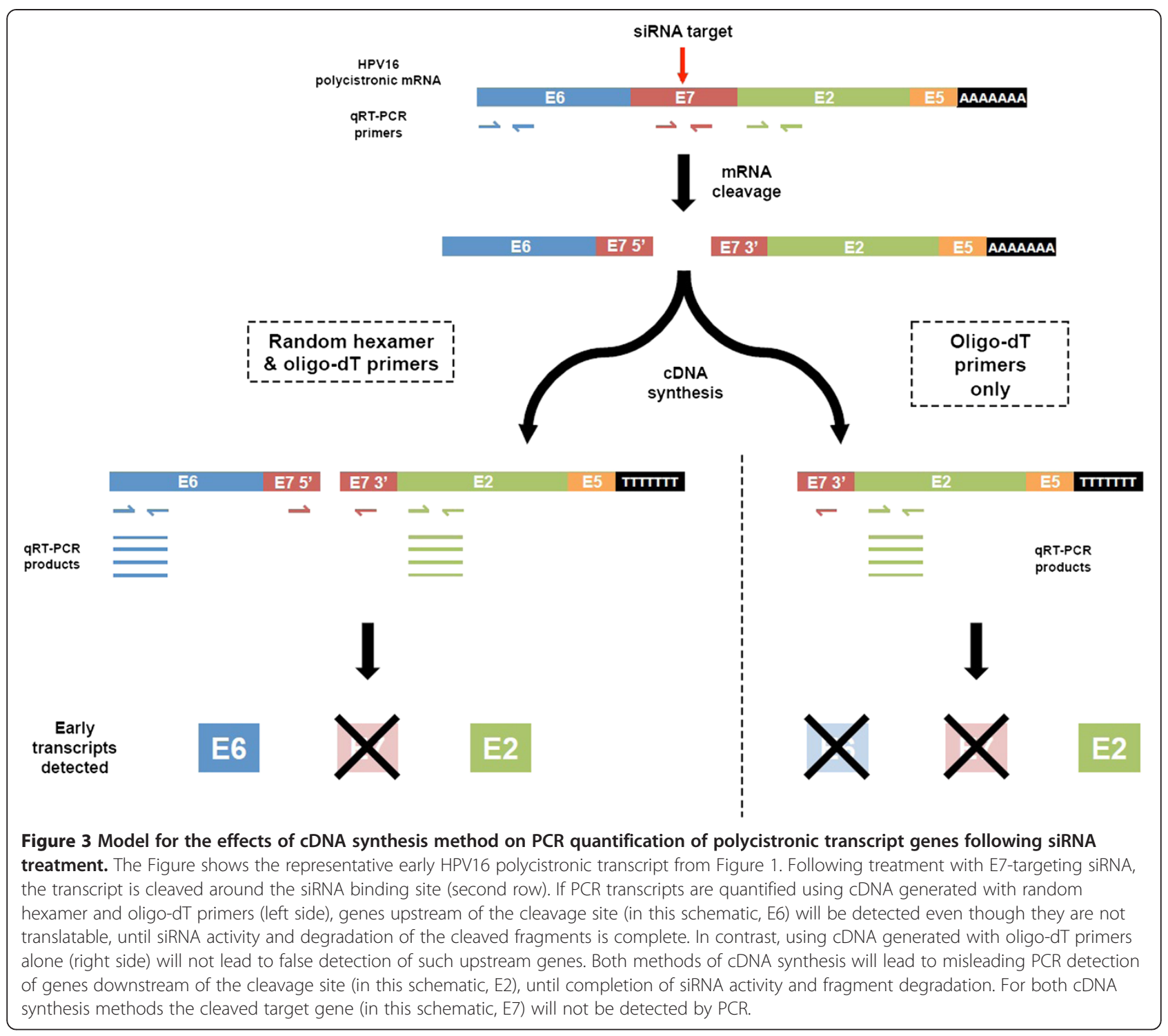

Our data may provide at least a partial explanation for conflicting observations in the literature concerning the specificity of siRNAs targeting HPV16 E7. The previous study that showed evidence of E7-specific effects [6] is likely to have quantified E6 in cleaved but undegraded mRNAs, as random decamers were included in the Reverse-iT reverse transcription kit used. It should be noted that differences in E6 versus E7 levels in the previous study persisted to 48 hours in CaSki and SiHa cells, in contrast to our present findings in W12. There was also continued suppression of p53 protein until 72 hours in cells treated with E7 siRNAs, although the presumption that this represented the sustained presence of E6 protein was not tested directly [6]. These caveats aside, our data suggest that when siRNAs are used to target any polycistronic mRNA transcript (i.e. not just those derived from HPV), careful consideration should be given to the method of cDNA synthesis and the relative binding sites of the PCR primers and siRNAs used.

\section{Competing interests}

The authors declare that they have no competing interests.

\section{Author contributions}

JEH carried out all molecular biology work, contributed to design of the study, was involved in all data analysis and interpretation and drafted the manuscript. IJG was involved in data analysis and manuscript preparation. MRP and NC conceived the study and participated in its design and interpretation of data analysis. NC co-wrote the manuscript. All authors read and approved the manuscript.

\section{Acknowledgements}

This study was funded by Cancer Research UK and the Medical Research Council.

Received: 20 February 2013 Accepted: 17 May 2013

Published: 21 May 2013 


\section{References}

1. Shah PS, Schaffer DV: Antiviral RNAi: translating science towards therapeutic success. Pharm Res 2011, 28:2966-2982.

2. Stanley MA: Human papillomavirus and cervical carcinogenesis. Best Pract Res Clin Obstet Gynaecol 2001, 15:663-676.

3. Zheng ZM, Baker CC: Papillomavirus genome structure, expression, and post-transcriptional regulation. Front Biosci 2006, 11:2286-2302.

4. Tang S, Tao M, McCoy JP, Zheng ZM: Short-term induction and long-term suppression of HPV16 oncogene silencing by RNA interference in cervical cancer cells. Oncogene 2006, 25:2094-2104.

5. Yamato K, Yamada T, Kizaki M, Ui-Tei K, Natori Y, Fujino M, Nishihara I, Keda Y, Nasu Y, Saigo K, Yoshinouchi M: New highly potent and specific E6 and E7 siRNAs for treatment of HPV16 positive cervical cancer. Cancer Gene Ther 2008, 15:140-153

6. Jiang M, Milner J: Selective silencing of viral gene expression in HPVpositive human cervical carcinoma cells treated with siRNA, a primer of RNA interference. Oncogene 2002, 21:6041-6048.

7. Elbashir SM, Harborth J, Lendeckel W, Yalcin A, Weber K, Tuschl T: Duplexes of 21-nucleotide RNAs mediate RNA interference in cultured mammalian cells. Nature 2001, 411:494-498.

8. Pattillo RA, Hussa R, Story M, Ruckert A, Shalaby M, Mattingly R: Tumor antigen and human chorionic gonadotropin in CaSki cells: a new epidermoid cervical cancer cell line. Science 1977, 196:1456-1458.

9. Hanning JE, Saini HK, Murray MJ, van Dongen S, Davis MP, Barker EM, Ward DM, Scarpini CG, Enright AJ, Pett MR, Coleman N: Lack of correlation between predicted and actual off-target effects of short-interfering RNAs targeting the human papillomavirus type $16 \mathrm{E7}$ oncogene. $\mathrm{Br} J$ Cance 2013, 108:450-460

10. Gray E, Pett MR, Ward D, Winder DM, Stanley MA, Roberts I, Scarpini CG, Coleman N: In vitro progression of human papillomavirus 16 episomeassociated cervical neoplasia displays fundamental similarities to integrant-associated carcinogenesis. Cancer Res 2010, 70:4081-4091.

doi:10.1186/1743-422X-10-159

Cite this article as: Hanning et al:: Depletion of polycistronic transcripts using short interfering RNAs: cDNA synthesis method affects levels of non-targeted genes determined by quantitative PCR. Virology Journal 2013 10:159.

\section{Submit your next manuscript to BioMed Central and take full advantage of:}

- Convenient online submission

- Thorough peer review

- No space constraints or color figure charges

- Immediate publication on acceptance

- Inclusion in PubMed, CAS, Scopus and Google Scholar

- Research which is freely available for redistribution 